ally, ten washings were sufficient to ensure complete removal of the cleaning solutions.

The more dilute solutions of soap and especially of hydrochloric acid give better results than concentrated ones, as described before ${ }^{1}$, because no close control of the time of contact between the grids and the cleaning solutions was necessary.

This method of cleaning used grids has some advantages over the flambage cleaning method proposed by Eaves ${ }^{2}$, because it does not make them friable nor does it destroy copper grids, as pointed out by Eaves; however, it does involve much more material and time.

$$
\text { Persio de Souza Santos }
$$

Laboratorio de Virus,

Instituto Butantan,

São Paulo, Brazil. Oct. 12.

${ }^{1}$ Souza Santos, P. de, Ciencia e Cultura (Sao Panlo), 4, 46 (1952). - Eaves, G., Nature, 169, 975 (1952).

\section{Use of Methyl Cellulose Gel as a Substitute for Agar in Tissue-Culture Overlays}

IN the course of experiments designed to assay non-cytopathogenic animal viruses by Dulbecco's plaque-count method ${ }^{1}$ using fluorescent antibody ${ }^{2}$ to identify the plaques, a non-fluorescent substitute was required for the agar used in the overlay. Methyl cellulose has been used by Earle ${ }^{3}$ to increase the viscosity of tissue-culture fluids for suspended-cell cultures, and appears to be harmless to animal tissue. An investigation of its properties showed it to be capable of forming highly viscous aqueous solutions which would gel spontaneously on raising their temperature. The presence of salts at physiological concentrations lowers the temperature of gelation. Thus one can make a solution of methyl cellulose containing tissue nutrients which, after being added as a liquid at room temperature to the tissue, will set quite firmly soon after being placed in an incubator at $37^{\circ} \mathrm{C}$. A further advantage is that this overlay can be removed entirely without disturbing the tissue; this is achieved by cooling the culture in a refrigerator for a short time, during which the gel liquefies, enabling it to be removed by pipetting.

The following procedure was used for obtaining 'bare' plaques of fowl-plague virus entirely free from overlay. Petri-dish tissue cultures of chick tissue monolayers were made by Dulbecco's method ${ }^{1}$, and infected ; $5 \mathrm{ml}$. of 1.5 per cent methyl cellulose overlay was poured on to each infected plate and incubation continued for a further three days. The overlay was prepared as follows : $1.5 \mathrm{gm}$. methyl cellulose of 4,000 centipoises viscosity (Dow Chemical Co.) (previously thoroughly washed with alcohol and ether) was added to $15 \mathrm{ml}$. de-ionized distilled water at $100^{\circ} \mathrm{C}$. in a 250 -ml. conical flask. The flask was shaken to disperse the methyl cellulose (which is insoluble at this temperature) and immediately autoclaved. After removal (still hot) from the autoclave, a warm sterile mixture of $5 \mathrm{ml}$. four-fold Earle's saline ${ }^{4}$ and $80 \mathrm{ml}$. synthetic medium (Waymouth, C., personal communication) containing antibiotics was added and thoroughly mixed. On cooling rapidly with agitation, the methyl cellulose dissolved to give a clear viscous solution. This was stored frozen at $-20^{\circ} \mathrm{C}$.

Following incubation of Petri-dish cultures, the overlay was pipetted off after adding $10 \mathrm{ml}$. of phosphate-buffered saline at $0^{\circ} \mathrm{C}$. to each plate, and chilling for $\frac{1}{2} \mathrm{hr}$. The cultures were rinsed with more phosphate saline and were then available for staining for $10 \mathrm{~min}$. with vital stain, or other treatment.

Under this overlay, plaques due to fowl plague virus take three days to become visible, whereas under agar only two days are required. This is believed to be due to a lower rate of diffusion of the virus in the methyl cellulose overlay; tests have indicated that the latter has no inhibitory or inactivating effect upon this virus.

Advantages of methyl cellulose overlay over agar are : (1) non-fluorescence; (2) simpler handling after a stock is made up ; (3) overlay can be completely removed ; (4) complete absence of toxicity on cells.

Disadvantages of the method are : (1) plaques take longer to form ; (2) stock solutions are more difficult to prepare; (3) gel is much weaker than that obtained with agar of equivalent concentration.

A few experiments have been made with methyl cellulose gel for other uses in tissue culture. It may be used as a substitute for plasma clot, but seems inferior to the latter as judged by the rate of increase in size of outgrowths from explants. Tests indicate that such gels have useful applications in bacteriology ; these are under investigation.

\section{J. E. Hotchin}

Medical Research Council

Common Cold Research Unit, Harvard Hospital,

Salisbury.

Oct. 7.

${ }^{1}$ Dulbecco, R., Proc. U.S. Nat. Acad. Sci., 38, 747 (1952).

${ }^{2}$ Coons, A. H., and Kaplan, M. IL., J. Exp. Med., 91, 1 (1950).

${ }^{3}$ Earle, W. R., Schilling, F. L., Bryant, J. C., and Evans, V. J., J. Nat. Can. Inst., 14, 1159 (1954).

4 Earle, W. R., J. Nat. Can. Inst., 4, 165 (1943).

\section{Preservation of Dogfish}

IN institutions where dogfish are used for dissection, the preservative most commonly employed is a solution of formaldehyde in water. Formaldehyde is a good preservative; but its volatile and pungent character makes its use in the class-room and the storeroom a matter of disagreeable compulsion.

Experiments first started in 1939 to find a suitable storage fluid to replace formaldehyde showed that pine oil compounds possessed many advantages in checking the growth of bacteria and fungi. 'Perminal KB' is a pine oil emulsion produced by Imperial Chemical Industries, Ltd., to assist in the kier boiling of cotton-as a solvent for fats and waxes, and as an emulsifying agent. When diluted with water it is an excellent preserving fluid for dogfish which have been fixed in formaldehyde. The steps are as follows: (1) wash the fixed dogfish in water overnight to remove the formaldehyde; (2) place in 'Perminal KB' 10 c.c., water 90 c.c.; rinse the specimens under the tap before dissection.

Dogfish so treated have been kept in this solution for more than five years without evidence of deterioration; but it should be emphasized that this method is of use only with fixed material. Fresh, unfixed material placed in this solution will decompose.

Zoology Department,

H. F. StEEDMAN

University, Glasgow, W.2. Nov. 1. 